\title{
Color Texture Discrimination using the Principal Geodesic Distance on a Multivariate Generalized Gaussian Manifold
}

\author{
Geert Verdoolaege ${ }^{1,2}$ and Aqsa Shabbir ${ }^{1,3}$ \\ 1 Department of Applied Physics, Ghent University, B-9000 Ghent, Belgium \\ geert. verdoolaege@ugent. be \\ 2 Laboratory for Plasma Physics - Royal Military Academy (LPP-ERM/KMS), \\ B-1000 Brussels, Belgium \\ 3 Max-Planck-Institut für Plasmaphysik, D-85748 Garching, Germany
}

\begin{abstract}
We present a new texture discrimination method for textured color images in the wavelet domain. In each wavelet subband, the correlation between the color bands is modeled by a multivariate generalized Gaussian distribution with fixed shape parameter (Gaussian, Laplacian). On the corresponding Riemannian manifold, the shape of texture clusters is characterized by means of principal geodesic analysis, specifically by the principal geodesic along which the cluster exhibits its largest variance. Then, the similarity of a texture to a class is defined in terms of the Rao geodesic distance on the manifold from the texture's distribution to its projection on the principal geodesic of that class. This similarity measure is used in a classification scheme, referred to as principal geodesic classification (PGC). It is shown to perform significantly better than several other classifiers.
\end{abstract}

Keywords: texture classification, Rao geodesic distance, principal geodesic analysis

\section{Introduction}

Texture discrimination is an essential task in various image processing applications, such as image retrieval and image segmentation. Texture is often characterized by means of the distribution of filter responses. In [1] the Rao geodesic distance (GD) based on the Fisher-Rao metric tensor was proposed as a similarity measure between multivariate generalized Gaussian distributions (MGGDs) characterizing the wavelet detail features of color textures. Among other advantages, it turns out that, for fixed shape parameter, an analytic expression exists for the GD on the MGGD submanifold, in contrast to the Kullback-Leibler divergence (KLD), barring the two-dimensional case [2]. Moreover, in [2] it was shown that, compared to the KLD, the GD provides consistently superior performance in its application to various texture classification and retrieval experiments.

Texture discrimination techniques frequently compute the distance between the unlabeled (query) texture image and one or several of its nearest neighbors 
in the training set. However, they seldom take into account the underlying shape or variability of the class. When the features consist of distribution parameters, this may be done by characterizing the shape of the cluster on the corresponding probabilistic manifold. Provided the clusters are compact, the class centroid yields a convenient summary of the cluster, which may be sufficient to discriminate between the various classes, as was done in [3]. On the other hand, for non-compact clusters a more sophisticated measure of cluster shape is required. For this reason in [4] texture classes were modeled by multiple centroids in an eigenspace of distance matrices.

In this paper we take a different approach which hinges on the observation that clusters of MGGD dispersion matrices form elongated structures on the manifold. The elongation is typically very pronounced along one or a few directions at most. Therefore we choose to characterize the cluster shape intrinsically in terms of the cluster's geodesic subspaces obtained by principal geodesic analysis. We present a new scheme for texture discrimination on the zero-mean MGGD manifold with fixed shape. It is based on the geodesic distance between the unlabeled texture and its projection on the principal geodesic corresponding to the largest eigenvalue for each class. Using data from a challenging color texture database, we compare the performance of our proposed scheme, which we refer to as principal geodesic classification, with the performance of the GDbased $k$-nearest neighbour classifier and another strategy based on the GD to a single cluster centroid ('distance-to-centroid'). This paper builds on our earlier work in [5], but here we present more mathematical details about the method and the experiments have been extended significantly.

\section{The manifold of multivariate generalized Gaussian distributions}

In our application the wavelet detail coefficients of color textures are modeled by means of a zero-mean MGGD, considering the dependence between the color bands. The wavelet subbands are assumed to be mutually independent. We first introduce the MGGD model and then we discuss the geodesics, the exponential map and the Fréchet mean on the MGGD manifold. We assume that, where necessary, existence and uniqueness conditions are fulfilled.

\subsection{The multivariate generalized Gaussian distribution}

We adopt the definition of the zero-mean MGGD (or multivariate exponential power distribution) provided in [1], with the following density function for the vector $\boldsymbol{x}$ :

$$
f(\boldsymbol{x} \mid \Sigma, \beta)=\frac{\Gamma\left(\frac{m}{2}\right)}{\pi^{\frac{m}{2}} \Gamma\left(\frac{m}{2 \beta}\right) 2^{\frac{m}{2 \beta}}} \frac{\beta}{|\Sigma|^{\frac{1}{2}}} \exp \left[-\frac{1}{2}\left(\boldsymbol{x}^{\prime} \Sigma^{-1} \boldsymbol{x}\right)^{\beta}\right] .
$$

Here, $m$ is the dimensionality of the probability space, e.g. $m=3$ for three-band color images. Also, $\Gamma($.$) denotes the Gamma function and \Sigma$ is the dispersion 
matrix. $\beta$ is the shape parameter which controls the fall-off rate of the distribution. The multivariate Gaussian case is retrieved for $\beta=1$, while we refer to the case $\beta=1 / 2$ as the multivariate Laplace distribution. Owing to its heavier tails, the Laplace distribution is expected to provide a better model for wavelet statistics; a fact that was confirmed in earlier classification experiments [2]. In the experiments below, the parameters of the probability models were estimated via the method of moments, followed by an optimization through maximum likelihood estimation [2].

\subsection{Geodesic distance}

The geodesics for the zero-mean MGGD were derived in [1]. We here only consider the case with fixed shape parameter $\beta$, corresponding to a set of submanifolds, each parameterized by the dispersion matrix $\Sigma$. The dimensionality of each submanifold is given by $N=m(m+1) / 2$, resulting in $N=6$ dimensions for three-band color images. However, it turns out that the metric and geodesics assume a particularly simple form in another parameterization, obtained as follows [1]. First, we consider the geodesic between two specific dispersion matrices $\Sigma_{1}$ and $\Sigma_{2}$. Then, we calculate the regular matrix $K$ that simultaneously diagonalizes $\Sigma_{1}$ and $\Sigma_{2}$, sending $\Sigma_{1}$ to the unit matrix $I_{m}$ and $\Sigma_{2}$ to a diagonal matrix $\Phi_{2}$ :

$$
K^{\prime} \Sigma_{1} K=I_{m}, \quad K^{\prime} \Sigma_{2} K=\Phi_{2} .
$$

The diagonal elements of $\Phi_{2}$ are the eigenvalues $\lambda_{2}^{i}$ of $\Sigma_{1}^{-1} \Sigma_{2}(i=1, \ldots, m)$. With a final coordinate transformation to $r_{2}^{i} \equiv \ln \lambda_{2}^{i}$, the metric elements $g_{i j}$ are constants given by

$$
\begin{aligned}
& g_{i i}=3 b_{h}-\frac{1}{4}, \\
& g_{i j}=b_{h}-\frac{1}{4}, \quad i \neq j, \quad \text { where } \quad b_{h} \equiv \frac{1}{4} \frac{m+2 \beta}{m+2} .
\end{aligned}
$$

In fact it can be proved that $K$ diagonalizes all matrices $\Sigma(t)$ on the geodesic between $\Sigma_{1}$ and $\Sigma_{2}$, parameterized by $t(0 \leq t \leq 1)$. As such, $K$ reduces $\Sigma(t)$ to $\Phi(t)$, a diagonal matrix with elements the eigenvalues $\lambda^{i}(t)$ of $\Sigma_{1}^{-1} \Sigma(t)$, where $\lambda_{2}^{i} \equiv \lambda^{i}(1)$. The geodesic between $\Sigma_{1}$ and $\Sigma_{2}$ is then simply a straight line:

$$
r^{i}(t)=\ln \left(\lambda_{2}^{i}\right) t
$$

where $r^{i}(t) \equiv \ln \left[\lambda^{i}(t)\right]$. As a result, the geodesic distance between the two distributions becomes [1]

$$
\operatorname{GD}\left(\Sigma_{1}, \Sigma_{2}\right)=\left[\left(3 b_{h}-\frac{1}{4}\right) \sum_{i}\left(r_{2}^{i}\right)^{2}+2\left(b_{h}-\frac{1}{4}\right) \sum_{i<j} r_{2}^{i} r_{2}^{j}\right]^{1 / 2}
$$




\subsection{Exponential map}

The exponential map, sending tangent vectors to points on the manifold, as well as its inverse, will be needed for subsequent calculations. A tangent vector in the starting point $(t=0)$ of a geodesic provides a 'velocity vector' for that geodesic. In terms of the matrices $\Phi(t)$ the tangent vectors $T$ are given by

$$
T=\left.\frac{\mathrm{d} \Sigma(t)}{\mathrm{d} t}\right|_{t=0}=\left.\left(K^{\prime}\right)^{-1} \frac{\mathrm{d} \Phi(t)}{\mathrm{d} t}\right|_{t=0} K^{-1}=\left(K^{\prime}\right)^{-1} \ln \left(\Phi_{2}\right) K^{-1},
$$

where the last equality follows from (2). Clearly the tangent vector $T$ is also diagonalized by the same matrix $K$, resulting in the matrix $\ln \left(\Phi_{2}\right)$. Consequently, given a tangent vector and a point of application $\Sigma_{1}$ on the manifold, to find the exponential map of the tangent vector $T$ we merely need to calculate the matrix $K$ that diagonalizes both $\Sigma_{1}$ and $T$, sending $\Sigma_{1}$ to the unit matrix. The only remaining operation is the normalization, since we have to find the point on the geodesic that lies at a geodesic distance $\|T\|$ from $\Sigma_{1}$. From (3) and (4) it follows that rescaling the logarithmic eigenvalues $\ln \lambda_{2}^{i}$ by a factor $k$ also rescales the GD by the same factor. Therefore, after calculating the GD, resulting in a 'temporary' value of, say $\mathrm{GD}_{0}$, corresponding to the logarithmic eigenvalues $\ln \lambda_{2}^{i}$ obtained by diagonalization of $\Sigma_{1}$ and $T$, we simply need to rescale $\ln \lambda_{2}^{i}$ by a factor $\|T\| / \mathrm{GD}_{0}$. Then, the result of the exponential map $\Sigma_{2}$ applied to $T$ is given by

$$
\Sigma_{2}=\left(K^{\prime}\right)^{-1} \ln \left(\Phi_{2}\right) \frac{\|T\|}{\mathrm{GD}_{0}} K^{-1} .
$$

Conversely, to find the result of the inverse exponential map, or logarithmic map, taking a point $\Sigma_{2}$ to the tangent vector $T$ in $\Sigma_{1}$, with $\|T\|=\operatorname{GD}\left(\Sigma_{1}, \Sigma_{2}\right)$, we first calculate the following 'temporary' tangent vector:

$$
T_{0}=\left(K^{\prime}\right)^{-1} \ln \left(\Phi_{2}\right) K^{-1} .
$$

This still needs to be rescaled, resulting in the final image $T$ under the logarithmic map:

$$
T=T_{0} \frac{\operatorname{GD}\left(\Sigma_{1}, \Sigma_{2}\right)}{\left\|T_{0}\right\|} .
$$

\subsection{Fréchet mean}

The Fréchet or Kärcher mean provides a generalization to the manifold setting of the centroid of a cluster of points in a Euclidean space. Given a set of $n$ points $\Sigma_{j}$ on the fixed-shape zero-mean MGGD manifold, the centroid $\Sigma_{\mathrm{c}}$ is obtained through the following minimization:

$$
\Sigma_{\mathrm{c}}=\underset{\Sigma}{\operatorname{ArgMin}} \sum_{j=1}^{n} \operatorname{GD}^{2}\left(\Sigma, \Sigma_{j}\right)
$$

This poses an optimization problem on the manifold. Assuming that a solution exists and that it is unique, we solve the problem iteratively by projecting the 
points $\Sigma_{j}$ on the tangent space at the current approximation to the centroid (initialized by that $\Sigma_{j}$ which minimizes the criterion (5)). Then we calculate their Euclidean mean on the tangent space and project the result back to the manifold, as illustrated in Figure 1a. This is basically a gradient descent algorithm on the manifold, which was derived in [6].

\section{Principal geodesic classification}

The proposed principal geodesic classifier on the MGGD manifold is based on principal geodesic analysis (PGA). We briefly describe PGA in this section, followed by an outline of the principal geodesic classification (PGC) algorithm.

\subsection{Principal geodesic analysis}

Since a geodesic is in a sense a generalization of a straight line in a Euclidean space, PGA for a cluster of points on a manifold was proposed as a natural generalization of principal component analysis (PCA) [7]. PGA yields a set of nested submanifolds, on which the projected elongation or variance of the cluster is maximal. Approximating the projection on the subspaces by the inner product in the tangent space at the centroid, PGA can be carried out through PCA in the tangent space (exact PGA would computationally be too demanding). The resulting tangent vectors, which are the eigenvectors of the covariance matrix in the tangent space, uniquely define a set of geodesic subspaces of the manifold.

It is important to note that PGA yields an (approximately) intrinsic characterization of the cluster, which is certainly to be preferred over tangent space approximations in the case of elongated structures. For instance, in our experiments we noted that a classifier based on the Mahalonobis distance in the tangent space at each cluster centroid, did not yield satisfactory results. Although the issue was not studied in detail, it is possible that the reason lies in the distortion that occurs through the projection on the tangent space. Indeed, on geometrical grounds it is clear that, as a result of the distortion, the error on the Mahalanobis distance is generally larger for more elongated clusters.

\section{2 $\quad$ PGC training and testing}

The PGC training phase consists of providing the model for each class by means of PGA. In the experiments below, we retain only the first principal geodesic, characterizing the direction along which the cluster has its largest elongation or variance.

In the testing phase, each texture in the database is considered one after the other. Such a test (or query) texture is then projected on the first principal geodesic of each class. This in itself is an optimization problem, as it involves finding the point on the geodesic that has the shortest GD to the test point. As with PGA, the projected point could be approximated by performing the projection in the tangent space and taking the image under the exponential map. 
However, it will be shown that this noticeably reduces the overall performance of the classifier. Therefore it is better to carry out the exact projection through optimization in terms of the parameter $t$ along the principal geodesic. Subsequently, the GD is calculated between the test point and its projection on the principal geodesic. This principal geodesic distance is defined as our similarity measure between the test point and a class. This is illustrated in Figure 1b.

As the wavelet subbands are considered to be mutually independent, this procedure can be carried out in each individual subband. The total squared GD between the test point and the class is then taken as the sum of squared GDs in each individual subband. Finally, the test point is assigned to the class to which its total principal geodesic distance is the smallest.

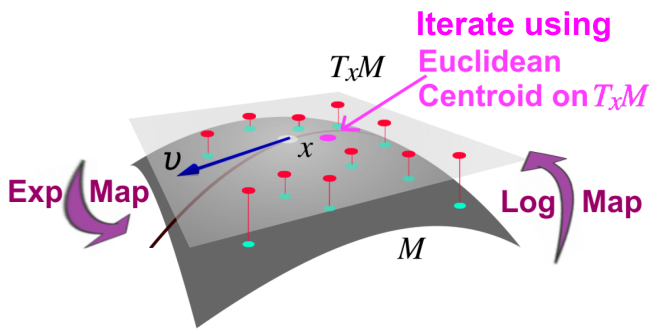

(a)

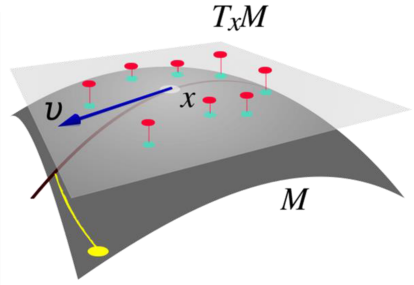

(b)

Fig. 1: (a) Principle of the iterative algorithm to calculate the centroid of a cluster on the MGGD manifold. (b) Illustration of classification of a test texture by PGC. For each class, the distance is calculated of the test texture to its projection on the first principal geodesic of that class.

\section{Classification experiments}

\subsection{Experimental setup}

An experiment was set up using data from the Columbia-Utrecht Reflectance and Texture Database (CUReT). It is characterized by a relatively large withinclass variability, leading to a highly challenging classification task. A subset of cropped $200 \times 200$ RGB images was chosen, belonging to 61 classes. Each class is made up of a single texture, imaged under varying illumination conditions and viewpoints [8]. As such, each class consists of 92 images, resulting in a database of 5612 images to be classified.

The class features were calculated as follows. Every color component of each image was individually normalized to zero mean and unit standard deviation. Then, a discrete wavelet transform with three levels and three orientations (nine subbands) was applied individually on every color component using the 
Daubechies filters of length eight. The wavelet detail coefficients of every subband were then modeled jointly over the three color components by an MGGD with $\beta=1$ (Gaussian) or $\beta=1 / 2$ (Laplacian). The resulting dispersion matrices constitute the feature set for a single image. Next, in all wavelet subbands the first principal geodesic was computed for each class of 92 images.

Finally, the classification was carried out based on the principal geodesic distance to each class. The classification performance was measured by the success rate using the leave-one-out strategy. We performed a comparison with a 'distance-to-centroid' (DtC) classifier that simply calculates the GD of the test texture to the centroid of each cluster. Another comparison was made with a $k$-nearest neighbor $(\mathrm{kNN})$ classifier. Here, $k=91$ was chosen since ideally the other 91 subimages should be the nearest neighbors of a test texture.

\subsection{Experimental results}

The results of the classification experiments are presented in Table 1. The highest classification accuracy is achieved with our proposed principal geodesic classifier, compared to the DtC classifier and $\mathrm{kNN}$. This indicates that accommodating the intrinsic variability of the texture classes on the MGGD manifold potentially leads to a performance improvement.

In addition, the Laplace distribution, for which the GD takes on a closed form, is indeed seen to perform better than the Gaussian distribution in most tests, and for PGC in particular.

It is also worth noting that the performance of the DtC classifier is inferior to that of kNN. Furthermore, it is the only case where the Laplace distribution performs worse than the Gaussian. This could indicate that, for this particular database, the characterization of the classes by means of a single centroid entails an excessive loss of information. We should also mention here that in earlier results on another database, the $\mathrm{DtC}$ scheme did yield considerably better results than the $\mathrm{kNN}$ classifier [3]. This remains a matter for further investigation.

Finally, PGC also offers a significant computational advantage over kNN. Indeed, although the training phase of PGC is more demanding, during classification $\mathrm{kNN}$ requires a distance calculation to each image in the database, while PGC merely needs the principal geodesic distance to each class. The computational advantage becomes even more pronounced when the approximation is employed whereby the projection onto the principal geodesic is performed in the tangent space.

\section{Conclusion}

We have presented a new classification scheme for color textures on a probabilistic manifold, exploiting the redundancy of the information in the parameters of the distribution to characterize the variability of texture classes. The multivariate generalized Gaussian distribution remains an interesting model for multiband wavelet features, particularly in view of the existence of an analytic expression for 
Table 1: Classification success rates (SR), based on Gaussian (G) and Laplace (L) models of 5612 CUReT color textures for three wavelet scales, using PGC (exact projection and approximation in the tangent space). This is compared to a distance-to-centroid (DtC) and a $k$-nearest neighbor $(\mathrm{kNN})$ classifier.

\begin{tabular}{lcccccccc}
\hline Classifier & \multicolumn{2}{c}{ PGC exact } & \multicolumn{2}{c}{ PGC approx. } & \multicolumn{2}{c}{ DtC } & \multicolumn{2}{c}{ kNN } \\
\hline Model & G & L & G & L & G & L & G & L \\
\hline SR & 80.6 & 82.5 & 76.6 & 77.6 & 72.3 & 69.8 & 73.5 & 75.7 \\
\hline
\end{tabular}

the Rao geodesic distance in the case of a fixed shape parameter. Our proposed principal geodesic classifier exhibits superior performance in a classification task on the CUReT texture database, in comparison with a distance-to-centroid and a $k$-nearest neighbor classifier.

Various avenues for future research have been identified, starting with existence and uniqueness conditions for the cluster centroids, projection onto the principal geodesic, etc. The weaker performance of the distance-to-centroid classifier in the present experiments is another issue to be investigated. Finally, projection on multiple geodesic subspaces along interesting directions would be a logical next development of the principal geodesic classifier.

\section{References}

1. G. Verdoolaege and P. Scheunders. On the geometry of multivariate generalized Gaussian models. J. Math. Imaging Vis., 43(3):180-193, 2011.

2. G. Verdoolaege and P. Scheunders. Geodesics on the manifold of multivariate generalized Gaussian distributions with an application to multicomponent texture discrimination. Int. J. Comput. Vis., 95(3):265-286, 2011.

3. A. Shabbir, G. Verdoolaege, and G. Van Oost. Multivariate texture discrimination based on geodesics to class centroids on a generalized Gaussian manifold. In Geometric Science of Information, volume 8085 of Lecture Notes in Computer Science, pages 853-860, Paris, France, 2013.

4. A. Schutz, L. Bombrun, and Y. Berthoumieu. K-centroids-based supervised classification of texture images using the SIRV modeling. In Geometric Science of Information, volume 8085 of Lecture Notes in Computer Science, pages 140-148, Paris, France, 2013.

5. A. Shabbir and G. Verdoolaege. Multivariate texture discrimination using a principal geodesic classifier. In IEEE International Conference on Image Processing, Québec City, Canada, September, 2015.

6. X. Pennec. Intrinsic statistics on Riemannian manifolds: basic tools for geometric measurements. J. Math. Imaging Vis., 25(1):127-154, 2006.

7. P.T. Fletcher, C.L. Lu, S.A. Pizer, and S. Joshi. Principal geodesic analysis for the study of nonlinear statistics of shape. IEEE Trans. Med. Imag., 23(8):995-1005, 2004.

8. Online at http://www.robots.ox.ac.uk/ vgg/research/texclass, 2008. 